\title{
Restoration of Images Corrupted by High Density Salt \& Pepper Noise through Adaptive Median Based Modified Mean Filter
}

\author{
R.Nisha ${ }^{1}$, P.Jeenath Nabela ${ }^{2}$, M.Sudheekshitha ${ }^{3}$, B.Madhusudhana Reddy ${ }^{4}$ \\ ${ }^{1,2,3,4}$ ECE,Vel Tech High Tech Dr.Rangarajan Dr. Sakunthala Engineering college, Chennai, India
}

\begin{abstract}
In this paper an efficient algorithm is proposed for removal of salt \& pepper noise from digital images. Salt and pepper noise in images is present due to bit errors in transmission or introduced during the signal acquisition stage. It represents itself as randomly occurring white and black pixels. This noise can be removed using standard Median Filter (SMF), Progressive Switched Median Filter (PSMF) under low density noise conditions. Decision Based Algorithm (DBA) and Modified Decision Based Unsymmetric Trimmed Median Filter (MDBUTMF) do not give better results at high noise density. So, in this project, this drawback will be overcome by using Adaptive Median based Modified Mean Filter (AMMF). This proposed algorithm shows better Peak Signal-to-Noise Ratio and clear image than the existing algorithm.
\end{abstract}

Keywords-Median filter, Progressive Switched Median Filter, Decision Based Algorithm, Modified Decision Based Unsymmetric Trimmed Median Filter

\section{Introduction}

Image noise is a random variation of brightness or color information in images. It can be produced by the sensor and circuitry of a digital camera, during image acquisition and transmission and faulty switching. This noise leads to blurring of image and loss of image details such as clarity. It can corrupt the images where the corrupted pixel takes either maximum or minimum gray level. There are two types of impulse noise, they are salt and pepper noise and random valued noise. Salt and pepper noise can corrupt the images where the corrupted pixel takes either maximum or minimum gray level. Several nonlinear filters have been proposed for restoration of images contaminated by salt and pepper noise. Among these SMF is a non-linear process useful in reducing salt and pepper noise. It is also useful in preserving edges in an image while removing noise. It replaces the value of a pixel by the median of the gray levels in the neighborhood of that pixel [1]. However, a major drawback of SMF is that the filter is effective only at low noise densities; its performance is poor when the number of noise pixels in the window is greater than half the number of pixels in the window and some median algorithms are not good for real time processing [2].

In PSMF, the decision is based on a predefined threshold value [3]. The algorithm is developed based on the following two main points: Switching scheme and Progressive methods. The major drawback of this method is that defining a robust decision is difficult and when a noise level is high, the edges may not be recovered satisfactorily. To overcome the above drawback, DBA is proposed [4]. In this, the noisy pixel can be removed either by the median value or by the mean of the previously processed neighboring pixel values. At high noise density the median value will be 0 or 255 which is noisy. In such case, neighboring pixel is used for replacement. This repeated replacement of neighboring pixel produces streaking effect. To avoid the major drawback of DBA, MDBUTMF is proposed. In this algorithm the noisy image is denoised by using " 3 X 3 " window elements and then pixels are arranged in increasing or decreasing order. Then the pixel values ' 0 's and ' 255 's in the image (i.e., the pixel values responsible for the salt and pepper noise) are removed from the image. Then the median value of the remaining pixels is taken. This median value is used to replace the noisy pixel [5]. This algorithm does not give better results at high noise density ranging from $70 \%$ to $95 \%$. So to overcome the drawback of MDBUTMF algorithm, a new \& efficient algorithm is proposed which is suitable for elimination of high density impulse noise ranging from $60 \%$ to $95 \%$.

The proposed AMMF algorithm consists of two stages: in first stage the noisy image is processed by adaptive median filter and in the second stage the output of first stage is further processed by modified mean filter. The proposed MDBUTMF algorithm removes this drawback at high noise density and it gives better Peak Signal-to-Noise Ratio (PSNR) values than the existing algorithm. This paper is organized in the following manner. Section 2 briefly describes about proposed algorithm, its performance is described in section 3 and section 4 gives the conclusion of paper.

\section{Proposed AMMF algorithm}

The proposed AMMF algorithm consists of two stages, in first stage the noisy image is processed by adaptive median filter. In second stage the output of first stage is further processed by modified mean filter if the image is corrupted by high density salt \& paper noise. The first stage classifies pixels as noise by comparing 
each pixel to its neighborhood pixels. The size of the neighborhood is adjustable. A pixel is considered noisy when it is different from majority of its neighborhood pixels. These noisy pixels are replaced by the median value of neighborhood pixels. Further the second stage also works in two steps: in the first step the noisy pixels are detected and in the second step each noisy pixel are replaced by the mean of noise free pixel of $2 \times 2$ window.

\subsection{Noise Detection in Modified Mean filter}

In this section the main purpose is to identify the "noisy pixel" and "noise free pixels". It is described as follows: We assume that the two intensities that present the impulse noise are the maximum and the minimum values of the images dynamic range (i.e. 0 and $L-1)$. Thus, in this stage, at each pixel location (x,y) ,we mark the mask $\alpha$ by using the equation (1)

$$
\alpha(x, y)=\left\{\begin{array}{lr}
1 \text { for } g(x, y)=L-1 \\
1 \quad \text { for } g(x, y)=1 \\
0 \quad \text { otherwise }
\end{array} \ldots \ldots \ldots \ldots \ldots . . . .\right.
$$

Where the value ' 1 ' indicates noisy pixel and the value ' 0 ' indicates the noise free pixel

2.2. Implementation of AMMF Algorithm

The proposed algorithm involves the following steps to remove the impulse noise:

Stage-I:

Step(1). Initialize the window size (maximum value of window size is 13X13) of the filter.

Step(2). Check if centre pixel is noisy in selected window, if YES then go to step 3. Otherwise move centre of window to next pixel and redo step 2.

Step(3). Find the value of $Z_{\min }, Z_{\max }$ and $Z_{\text {med }}$ in the selected window.

Step(4). Determine if $Z_{\text {med }}$ is noisy by $Z_{\text {min }}<Z_{\text {medn }}<Z_{\text {max }}$. If it holds, $Z_{\text {med }}$ is noise free pixel and jump to step 6 . Otherwise, $Z_{\text {med }}$ is noisy and go to step 5 .

Step(5). Increase window size and go back to Step 3.

Step(6). Replace the centre pixel with $Z_{\text {med }}$.

Step(7). Reset window size and move the centre of window to next pixel.

Step(8). Repeat the steps until all pixels are processed.

At high noise density of salt and paper noise, some of pixels are still noisy in stage-I which are further removed by passing through the entire image by stage II algorithm.

Stage-II:

Step(i). Initialize the window size of the filter by $2 \times 2$ window.

Step(ii). Find out the noise free pixels present in $2 \times 2$ window.

Step(iii). Find out the mean value of the noise free pixels in selected window.

Step(iv). Replace the noisy pixel by the calculated mean value in step (iii).

Step(v). Repeat steps from (i)- (iv) to process the entire image for removal of Salt \& Pepper Noise.

\section{Performance of AMMF algorithm}

In order to demonstrate the performance of AMMF algorithm, it is tested on different gray scale natural images (i.e. 8-bit/pixel) with the noise density (N.D.) ranging from $10 \%$ to $95 \%$. The AMMF algorithm gives better result as compared to SMF, centre weighted median filter (CWMF) [6], PSMF, open-close sequence filter (OCSF), DBA, MDBUTMF. Each time the test image is corrupted by salt \& pepper noise of different noise densities ranging from $10 \%$ to $95 \%$. The performance of AMMF algorithm is expressed in terms of PSNR and mean squared error (MSE).The PSNR estimates the quality of a reconstructed image with respect to an original image. Reconstructed images with higher PSNR are better. PSNR is defined in dB in equation (2).

$$
\text { PSNR }=10 \times \log _{10}\left[\frac{255^{2}}{\mathrm{MSE}}\right]
$$

Where MSE is mean squared error between original image (x) and denoised image $(\hat{x})$ which is given by equation (3)

\subsection{Experiment 1}

$$
\operatorname{MSE}=\frac{1}{N_{1} \times N_{2}} \sum_{i=1}^{N_{1}} \sum_{j=1}^{N_{2}}(x(i, j)-\hat{x}(i, j))^{2}
$$

Table 1 and Table 2 demonstrate the comparison of PSNR and MSE values of different filters respectively for gray scale House image with N.D ranging from 10\% to 95\%. The performance of AMMF algorithm is compared with various existing techniques such as SMF, PSMF, DBA, and MDBUTMF. It can be noticed from Table1 and Table 2 that AMMF algorithm, gives better result in comparison to other existing techniques in terms of PSNR \& MSE for low level noise density as well as high level noise density. Fig. 1 illustrates the graphical comparison of PSNR performance metric for different denoising algorithms for House. Fig. 2 illustrates the graphical comparison of MSE performance metric for different denoising algorithms for House. 


\subsection{Experiment 2}

In order to demonstrate visual enhancement of AMMF algorithm, another experiment is conducted on House image with N.D ranging from $80 \%$ to $95 \%$. The visual enhancement of AMMF algorithm is compared with various existing techniques such as SMF, PSMF, DBA, and MDBUTMF. The visual enhancement of House \& Lena images are shown in Fig. 5(B), 5(C), 5(D), 5(E), 5(F), 6(B), 6(C), 6(D), 6(E), 6(F), 6(G), 6(H), Fig. 7(B), 7(C), 7(D), 7(E), 7(F), with noise density (N.D.) i.e. $0.80,0.90$ and 0.95 respectively. It is clear from Fig. 5 to Fig. 7 that the image recovered from the AMMF algorithm is better than other noise removal algorithms in terms of visibility.

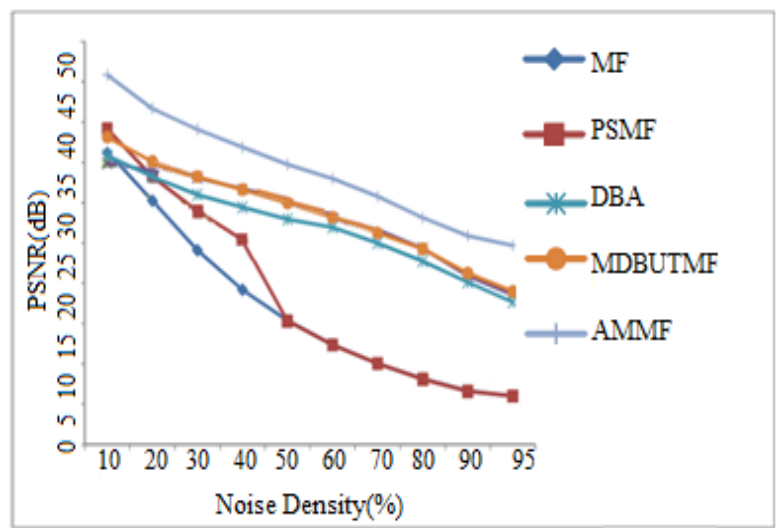

Fig.1. PSNR vs Noise Density for House Image

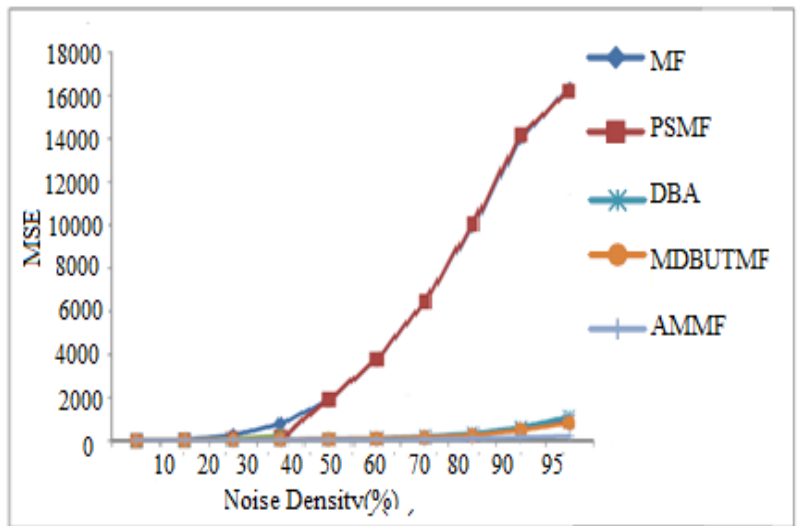

Fig.2. MSE vs Noise Density for House Image

\section{FLOWCHART OF AMMF:}

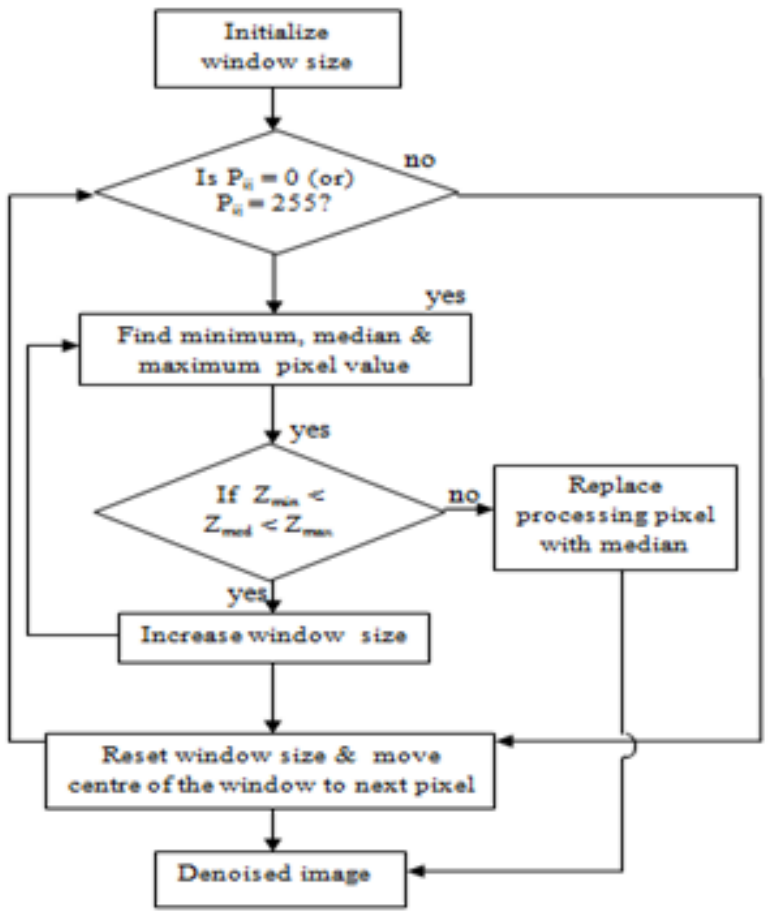

Stage I: Processing of noisy pixel using median filter

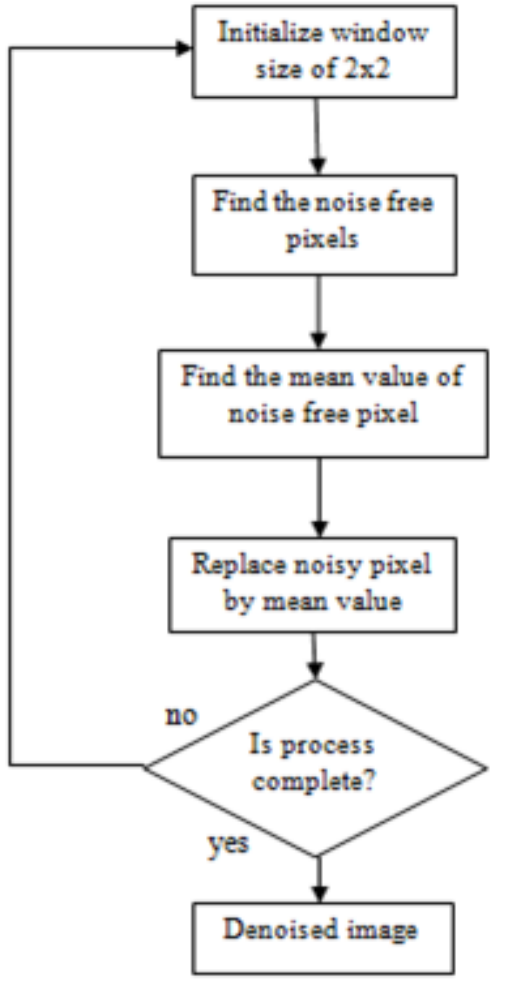

Stage II:Processing of noisy pixel using mean filter

\section{Conclusion}

This paper proposed a new algorithm (AMMF) for removal of salt \& pepper noise from digital images. The AMMF algorithm has been tested on different gray scale natural images with noise density ranging from $10 \%$ to $95 \%$. The performance of AMMF algorithm has been evaluated and compared in terms of PSNR and MSE values. The performance of AMMF algorithm has been compared with various existing techniques such as SMF, PSMF, DBA, MDBUTMF. Both visual and quantitative results are demonstrated. The AMMF algorithm 
Restoration of Images Corrupted by High Density Salt \& Pepper Noise through Adaptive Median

demonstrated well in low level noise density as well as high level noise density. Even at $95 \%$ noise density levels the proposed algorithm provided better results in comparison with other existing algorithms.

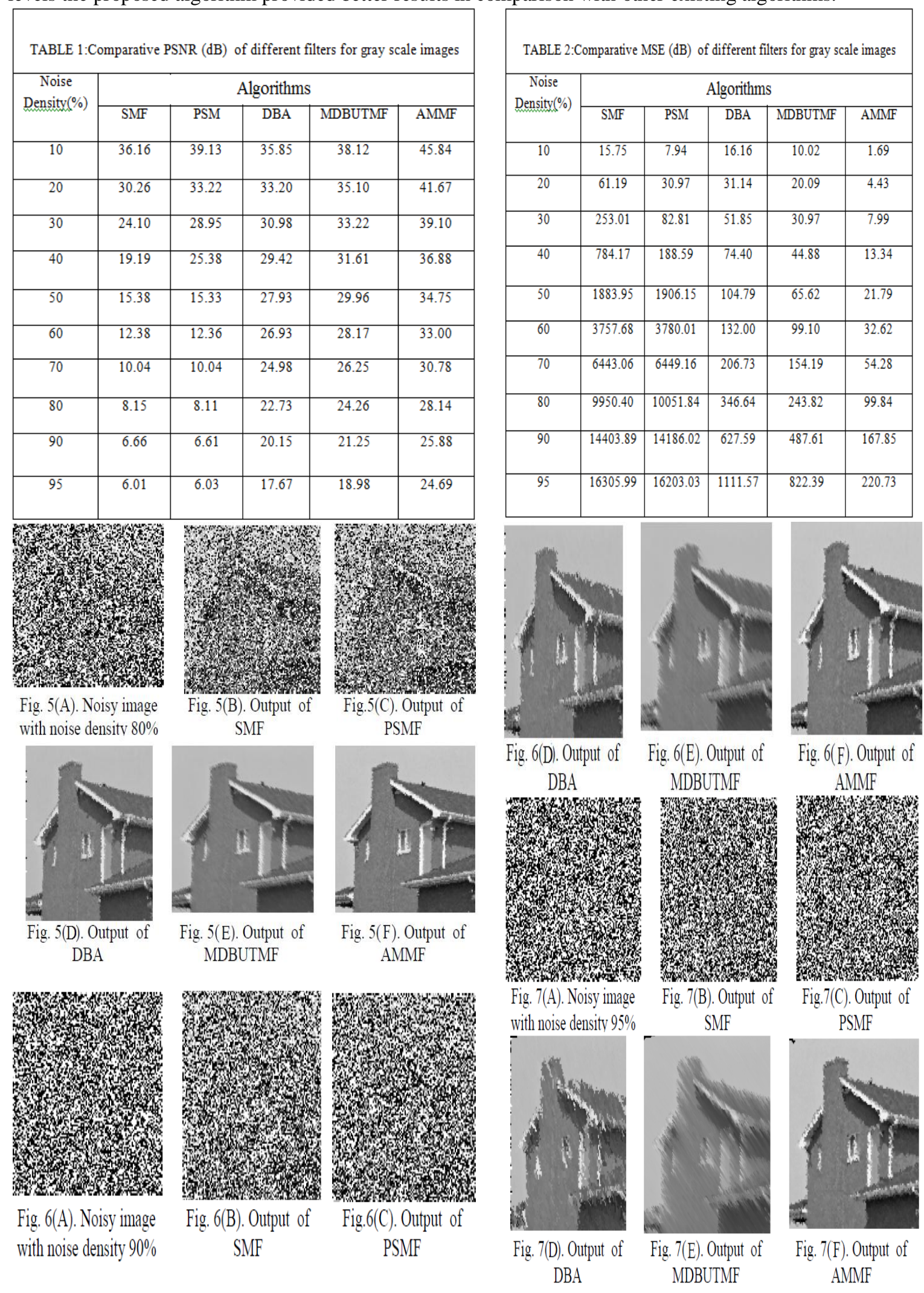




\section{Journal Papers:}

\section{REFERENCES}

[1] N.C Gallagher Jr and G.W. Wise, A theoretical analysis of the properties of median filter, IEEE Transactions on Acoustics, Speech, Signal Processing, vol. ASSP-27, 1981, pp.1136-1141.

[2] T. Huang, G. Yang, and G. Tang, A fast two dimensional median filtering algorithm, IEEE Transactions on Acoustics, Speech, Signal

[3] Z. Wang and D. Zhang, Progressive switching median filter for the removal of impulse noise from highly corrupted images, IEEE Transactions on Circuits and Systems-II, vol. 46, 1999, pp. 78-80.

[4] K. S. Srinivasan and D. Ebenezer, A new fast and efficient decision based algorithm for removal of high density impulse noise, IEEE Signal Processing Letters, vol. 14, no. 3, 2007, pp. 189-192.

[5] V. Jayaraj and D. Ebenezer, A new switching based median filtering scheme and algorithmfor removal of high-density salt and pepper noise in image, EURASIP Journal Advanced Signal Processing, vol. 2010, February 2010.

[6] T. Chen and H. R. Wu, Adaptive impulse detection using centre weighted median filters, IEEE Signal Processing Letters, vol. 8 , no. 1, 2001, pp. 1-3.

Books:

[7] R. C. Gonzalez, and Woods R.E, Digital Image Processing. (Addison-Wesley, Boston, 2005.) 